\title{
Simultaneous wetting and drying; fluid bed granulation and tablet film coating
}

Kemp, I.C. ${ }^{a^{*}}$; van Millingen, A. ${ }^{\text {a }}$ Khaled, H. ${ }^{\text {a }}$; Iler, L. ${ }^{b}$

${ }^{a}$ GSK, David Jack Centre for R\&D, Park Road, Ware, Hertfordshire, SG12 0DP, U.K.

b GSK, Global Pharma Manufacturing, Zebulon, North Carolina, U.S.A..

*E-mail of the corresponding author: ian.c.kemp@gsk.com

\begin{abstract}
Simultaneous wetting and drying occur in processes such as fluid bed (top spray) granulation, Wurster coating and tablet film coating. This gives control challenges, as the spraying and evaporation processes must be carefully balanced and the operating window is significantly narrower than for standalone drying processes. Significant recent advances in modelling have led to effective scale-up and operational strategies. Factors such as flow cessation during filter bag shaking can have a major effect. A design space can be predicted which is often non-orthogonal, and pharmaceutical regulatory authorities have accepted filing submissions using a design space justified by mechanistic modelling.
\end{abstract}

Keywords: pharmaceuticals; peak moisture content; bed temperature; conduction; design space. 


\section{Introduction}

Most drying processes involve drying of a pre-wetted material, either a wet solid liquid or a liquid solution/suspension as in spray drying. However, a few processes involve simultaneous wetting and drying of the solids. This gives significant effects on the drying behavior, and additional challenges result in modelling, design and practical operation. This paper focuses on two processes of this type, both employed in the pharmaceutical industry: fluid bed (top spray) granulation and tablet film coating. Both have been previously described in texts such as Pandey and Bharadwaj [1] and Lyngberg et al. [2]. However, modelling of these processes has been relatively neglected compared to standalone drying processes, even though successful operation is only possible within a narrower operating range, particularly for fluid bed granulation, as shown below. Recent advances in modelling and process understanding have led to improved methods for design, scale-up and operation, and are described in this paper. Applications in the pharmaceutical industry are described, in particular the development of an appropriate Design Space and operational ranges.

\section{Fluid bed granulation}

In fluid bed granulation, binder solution is sprayed on to a fluidized bed of particles and evaporated off simultaneously. The initial mixture of powders is gradually transformed into granules held together by solid bridges. Normally, the bed moisture content (LOD, loss-ondrying) increases gradually during spraying and then falls during a final drying phase, as in Fig. 1. However, if the solution spray rate is too fast, uncontrolled agglomeration can result, with potential adverse effects both on product quality (dissolution or content uniformity) and operation (partial or complete defluidisation, and potential loss of the batch).

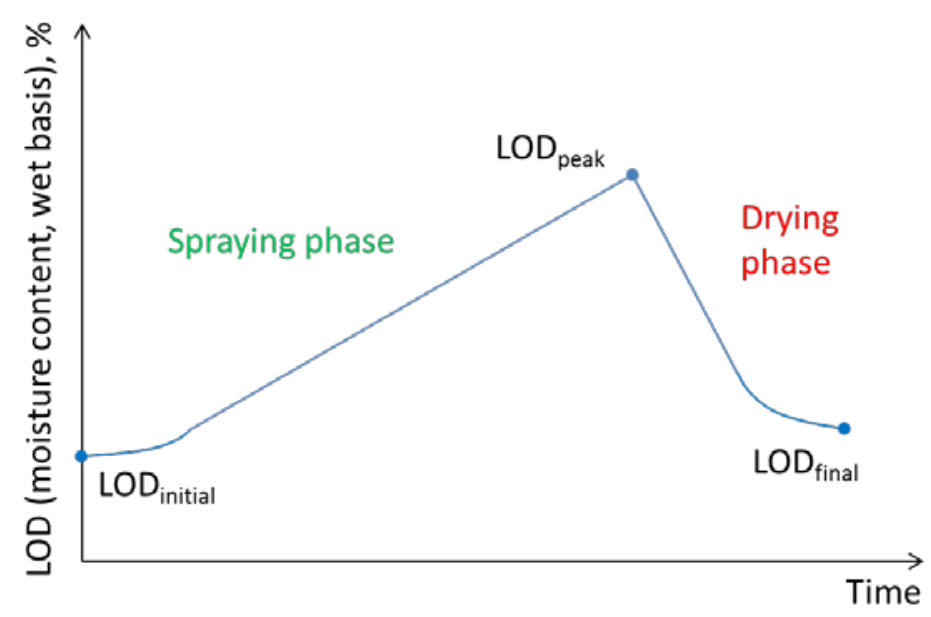

Fig. 1 Moisture content (LOD) during fluid bed granulation cycle. 


\subsection{Modelling - basic}

The rate of moisture accumulation, and hence the peak LOD, can be calculated using a heat and mass balance. The fundamental approach was described by Gupta [3], and a similar model was presented by Lyngberg et al [2]. The water accumulation rate in the granules, $m_{\text {accu }}(\mathrm{kg} / \mathrm{h})$, is the difference between the water in the incoming spray and that removed by evaporation:

$$
m_{a c c u}=m_{s o l}\left(1-x_{b}\right)-\left(\frac{Q_{a i r}-Q_{\text {loss }}}{h_{f g}}\right)
$$

Here, $m_{\text {sol }}=$ solution spray rate $(\mathrm{kg} / \mathrm{h}), x_{b}=$ binder concentration as mass fraction $(\mathrm{kg} / \mathrm{kg})$, $h_{f g}=$ latent heat of evaporation $(\mathrm{kJ} / \mathrm{kg}), Q_{\text {loss }}=$ heat loss rate $(\mathrm{kJ} / \mathrm{h})$, and $Q_{\text {air }}(\mathrm{kJ} / \mathrm{h})$ is the heat released from the air, defined in (2):

$$
Q_{a i r}=m_{a i r} C_{p, a i r}\left(T_{\text {in }}-T_{\text {out }}\right)=F_{a i r} \rho_{a i r} C_{p, a i r}\left(T_{\text {in }}-T_{\text {out }}\right)
$$

Where $\rho_{\text {air }}=$ air density $\left(\mathrm{kg} / \mathrm{m}^{3}\right), C_{p, \text { air }}=$ specific heat capacity of air $(\mathrm{kJ} / \mathrm{kgK})$.

Equations (1) and (2) show that four significant parameters affect the energy balance;

- $\quad$ Solution spray rate $\left(m_{\text {sol }}, \mathrm{kg} / \mathrm{h}\right)$

- Inlet air volumetric flow rate $\left(F_{\text {air }}, \mathrm{m}^{3} / \mathrm{h}\right)$ or mass flow rate $\left(m_{\text {air }}, \mathrm{kg} / \mathrm{h}\right)$

- Inlet air temperature $\left(T_{i n},{ }^{0} \mathrm{C}\right)$

- $\quad$ Outlet air temperature $\left(T_{\text {out }},{ }^{0} \mathrm{C}\right)$.

In fluid bed drying or granulation, if the particle surfaces remain fully wetted, the outlet air is close to saturation conditions, and is approximately equal to the wet bulb temperature $T_{w b}$ $\left({ }^{0} \mathrm{C}\right)$, which depends only on inlet air temperature and inlet air humidity $Y_{\text {in }}(\mathrm{g} / \mathrm{kg})$. Hence the four operating parameters which can affect heat input $Q_{\text {air }}$ are $m_{\text {sol }}, F_{\text {air }}, T_{\text {in }}$ and $Y_{\text {in }}$.

The mass accumulated is summed over the spraying period and can be transformed into a peak value of the loss on drying, LOD, which is a wet-basis moisture content:

$$
L O D_{\text {peak }}=\frac{M_{\text {sol }}}{M_{\text {bed,final }}}\left[\left(1-x_{b}\right)+\frac{Q_{\text {loss }}}{h_{f g} m_{\text {sol }}}-\frac{Q_{\text {air }}}{h_{f g} m_{\text {sol }}}\right]+\frac{M_{W, \text { initial }}}{M_{\text {bed, final }}}
$$

Where $M_{\text {bed,final }}$ is the total mass of the bed at the end of granulation (including dry solids, binder and moisture) and $M_{W \text {,initial }}$ is the total mass of water in the bed before granulation.

\subsection{Modelling - extended}

The standard model allows for heat losses, but Kemp et al [4] pointed out that several other factors affect the heat balance: 
i. Atomisation air flow from the two-fluid atomiser, normally at ambient temperature

ii. Additional heat from the initial preheated bed of solids

iii. Additional heat released when sprayed water becomes bound moisture

iv. Adjustments for periods when the spray or airflow are turned off or reduced, for example during filter bag shaking.

(i) can be handled by a small adjustment to $Q_{\text {air }}$ and (ii) and (iii) by small adjustments to $L O D_{\text {peak }}$, which like heat losses are best obtained by back-calculation from experimental measurement, and are the cause of the initial flatter section in the moisture-time curve. However, (iv) can have a significant effect. Exhaust air filters may operate with either "simultaneous" or "consecutive" cleaning. In consecutive cleaning, only a proportion of the filter bags are shaken or reverse pulsed at any moment, and the air flow and spray both continue. However, in simultaneous cleaning, all the filter bags are shaken at the same time, and air flow and spray are usually stopped. Typically, the spray is stopped, the airflow is ramped down, the bag is shaken, the airflow is restarted and finally the spray is restarted. Hence the spray is off for a longer period than the airflow and the balance between heat input and evaporation is changed. We can define fractional times $\tau_{\text {spray }}$ and $\tau_{\text {air }}$ as the proportion of the cycle that the water and air are flowing. Equation (3) now becomes:

$$
L O D_{p e a k}=\frac{M_{\text {sol }}}{M_{\text {bed, final }}}\left[\left(1-x_{b}\right)+\frac{Q_{\text {loss }}}{m_{\text {sol }} h_{f g} \tau_{\text {spray }}}-\frac{Q_{a i r} \tau_{a i r}}{m_{\text {sol }} h_{f g} \tau_{\text {spray }}}\right]+\frac{M_{W, \text { initial }}-M_{\text {corr }}}{M_{\text {bed, final }}}
$$

Where $M_{\text {corr }}(\mathrm{kg})$ is a correction factor allowing for the preheating and binding effects.

For a given granulation formulation and equipment, most of the terms in equation (4) are fixed or nearly constant, and the only two which depend on operating parameters are $Q_{\text {air }}$ and $m_{\text {sol }}$. Hence, peak LOD is predicted to vary with the ratio $\left(Q_{a i r} / m_{s o l}\right)$. From (1) and (4), high values of $Q_{a i r} / m_{\text {sol }}$ will give less accumulation and low peak LOD; conversely, low $Q_{a i r} / m_{\text {sol }}$ gives more accumulation and may give an excessive peak LOD.

Therefore, we can verify whether the mechanistic model fits well to a set of experimental data by plotting peak LOD against $Q_{a i r} / m_{\text {sol }}$. The expected slope and intercepts for peak LOD can be evaluated from equation (4).

\subsection{Design space}

Historically, operating conditions for pharmaceutical processes were often defined as a single set point, which was inconvenient and inflexible. The modern approach as defined in ICH Q8 [5] is to define a set of ranges for each key parameter, creating a "Design Space". The process can be operated anywhere within this envelope, but during development, it must be verified that the process can achieve key quality parameters under all possible operating conditions. 
The analysis above shows that four operating parameters, $m_{\text {sol }}, F_{\text {air }}, T_{\text {in }}$ and $Y_{\text {in }}$, can affect $Q_{a i r} / m_{\text {sol }}$ and hence peak LOD. It is convenient to represent these as a two-dimensional design space by combining the first 3 parameters into $Q_{\text {air }}$ (see Fig. 3 for an example). However, $Q_{a i r} / m_{\text {sol }}$ plots as sloping lines on this diagram, and is a minimum at the bottom right-hand corner, giving a maximum value for the peak LOD. If this leads to overwetting and defluidisation, this region will not be acceptable for operation. However, if the maximum peak LOD for successful granulation is found by experiment, the corresponding value of $Q_{a i r} / m_{\text {sol }}$ can be calculated and the available design space can be maximized by truncating the bottom right-hand corner. Likewise, if the top left-hand corner is too dry and gives poor granulation, this can also be truncated.

\subsection{Application to a real pharmaceutical process}

The model was applied to a real process for which experiments showed that stable granule size was obtained with a peak LOD of $12.2 \%$ or less [4]. Experimental data for peak LOD at both pilot-plant and commercial scale fitted well to a straight line when plotted against $Q_{\text {air }} / m_{\text {sol }}$, as shown in Figure 2. However, there was a substantial offset between the two sets of data. Using standard scaling rules based on the basic model in section 2.1, the LOD at commercial scale was significantly higher than predicted, and defluidisation was observed on a batch under "wet” conditions (high spray rate, low temperature, low airflow). The extended model in section 2.2 showed that the adjustments for atomization, moisture binding, bed preheating and heat losses were small, but that there was a substantial difference between the filter bag shaking regimes at pilot and commercial scale. The ratio $\tau_{\text {air }} / \tau_{\text {spray }}$ was much greater at pilot scale, and when equation (4) was applied instead of (3), this correctly predicted the observed difference between pilot and commercial scale.

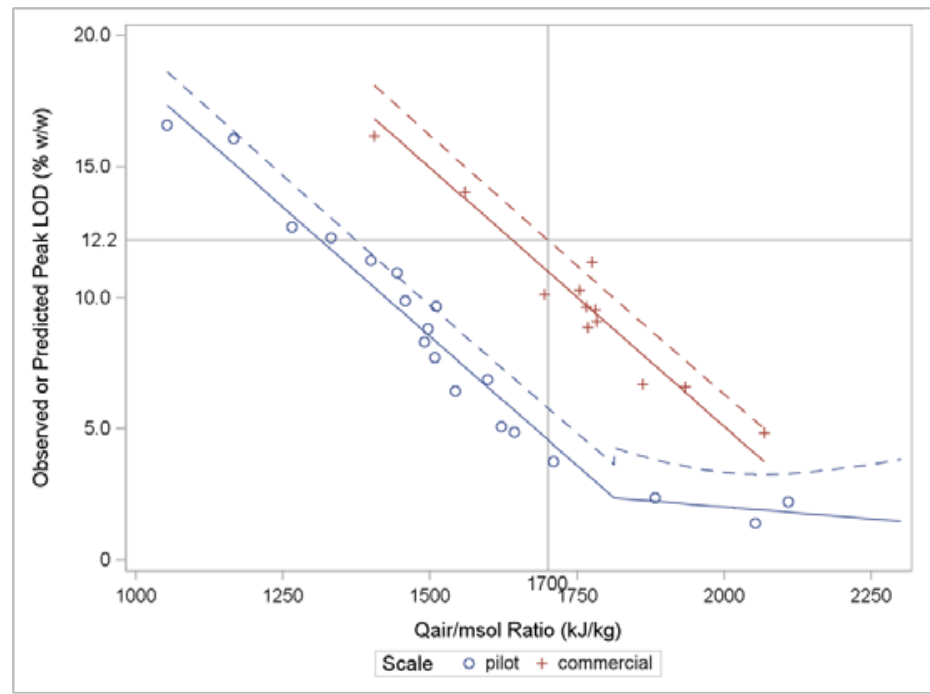

Fig. 2 Experimental results for peak LOD plotted against $Q_{a i r} / m_{\text {sol }}$ ratio 
The resulting commercial design space is shown in Figure 3. For operational simplicity, ranges can be defined for all the individual operating parameters, which plot as a rectangle which always falls within the design space. Moreover, the effect of changes in equipment can be anticipated, for example moving from a unit with simultaneous bag shaking (E1, broken lines) to consecutive shaking, where the air and liquid flows are continuous and $\tau_{\text {spray }}=\tau_{\text {air }}=1$ (E2, solid lines). The value of $Q_{\text {air }} / m_{\text {sol }}$ corresponding to the peak LOD is increased, the design space shrinks and the operating conditions need to be altered.

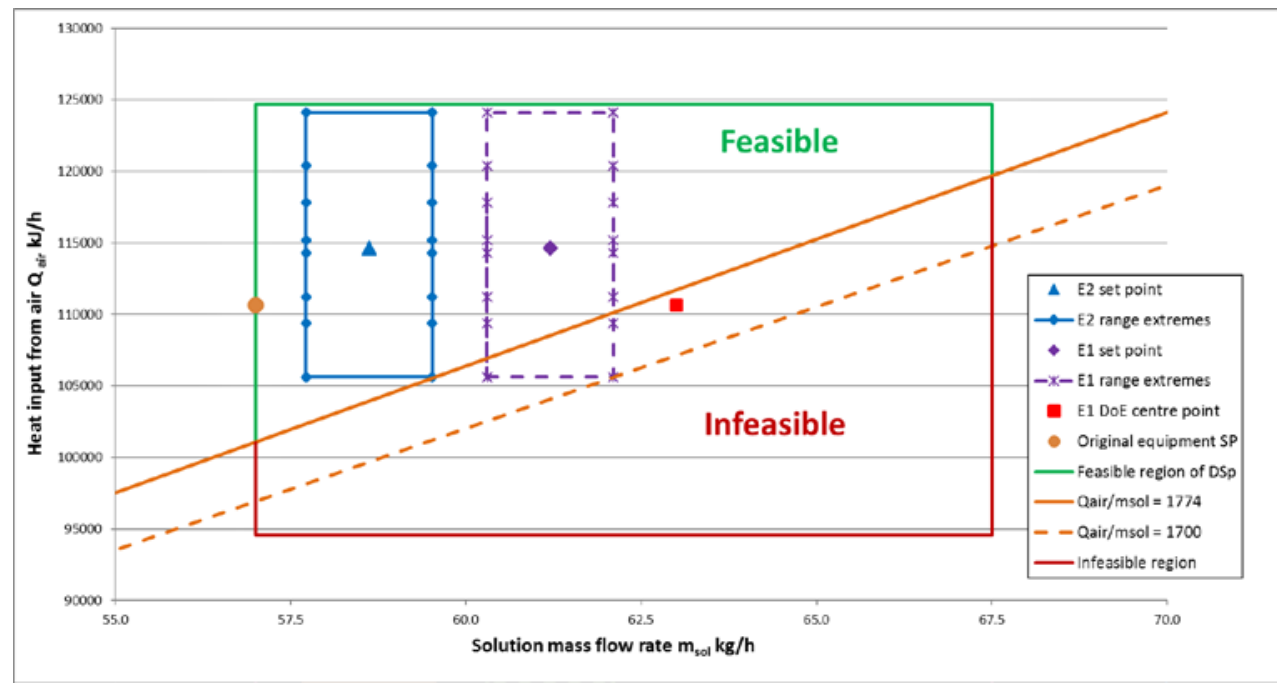

Fig. 3 Design and operating space at commercial scale with simultaneous bag shake and air cutoff (dotted lines) and consecutive bag shake with continuous flows (solid lines)

\section{Tablet film coating}

The most common type of pharmaceutical tablet coater is the perforated pan batch coater, in which coating solution is sprayed on to a region of the surface of a rolling bed of tablets. These tablets move back into the bulk bed within a few seconds and their surface must be sufficiently dry to avoid them sticking together, causing surface damage or "twinning”.

Current models were reviewed by Kemp et al [6], and can include four main aspects:

1. Mass balance - relates spray rate, suspension concentration and solids throughput

2. Heat balance - relates air flow rate, inlet and exhaust temperature and spray rate

3. Drying kinetics - calculates heat transfer and evaporation rates at the tablet surface

4. Spray effects - relates spray rate, spray area, coating film thickness and coating time.

The first two categories are routinely used in operation [7] - van den Ban et al. However, little had previously been done to study tablet surface kinetics, although Ebey [8] noted that 
heat and mass balances could not explain all observations and proposed an Environmental Equivalency (EE) factor to allow for this. Kemp et al [6] demonstrate that the locus of the boundary where overwetting and other adverse effects occur is dependent mainly on exhaust temperature and secondarily on spray rate. Again, this leads logically to the conclusion that if the size of the design space is to be maximized, it will have a sloping lower boundary. In practice, fixed operational ranges for exhaust temperature and spray rate will usually be more convenient, but the model also demonstrates that the key point where failure is most likely is the bottom right corner (low temperature, high spray rate). The modelling at tablet level also explains the long-standing observation that the bed temperature is significantly higher than the wet bulb temperature, unlike fluidised beds. At the tablet surface, the heat for evaporation is coming not primarily from the hot air, by convection, but by conduction from the warm tablet itself. The dry tablet bed is heated up by the air. For example, a coater with an air inlet temperature of $60^{\circ} \mathrm{C}$ will typically have a bed temperature of $40-45^{\circ} \mathrm{C}$ whereas the wet bulb temperature is $25^{\circ} \mathrm{C}$.

\section{Comparison between the processes}

Although fluid bed granulation and tablet film coating show many similar effects, there are some important conceptual differences. As noted above, in a fluidised bed of granules the heat of evaporation comes by convection from the hot air and the bed is normally at $T_{w b}$, whereas in a rolling bed of tablets the heat to evaporate the surface liquid film comes mainly by conduction from the tablet and the bed temperature is significantly above $T_{w b}$.

\subsection{Modelling}

Kemp [9] noted the distinction between models that can be "used once" (complex models to enhance process understanding) and "used regularly" (simple operational calculations). Both types of model are useful in these processes.

For fluid bed granulation, the full model can be "used once" for scale-up calculations and to establish the design space. A basic calculation of $Q_{a i r} / m_{\text {sol }}$ can be used to ensure that the everyday operating conditions remain within the design space.

For tablet coating, the full model demonstrates the effects of surface drying kinetics, the design space shape and the number of coating passes. For normal operation, the heat and mass balance is sufficient, e.g. to give new coating times if the coating type is changed (Opadry ${ }^{\circledR}$ II can work at higher concentrations than Opadry ${ }^{\circledR}$ I), or required inlet temperature for a given exhaust temperature and spray rate when developing a DoE.

\subsection{Design space}

The analysis shows that both processes can have a trapezoidal design space with a sloping lower boundary regulated by overwetting effects [8]. However, the governing factors are 
different. For fluid bed granulation the boundary line is given by the $Q_{a i r} / m_{\text {sol }}$ value which is dependent on the macroscopic heat and mass balance. In contrast, for tablet film coating the boundary is regulated by kinetic effects, balancing the incoming spray rate with the evaporation rate at the tablet surface due to the local tablet temperature, and the slope is given by the variation of vapour pressure with temperature.

\section{Conclusions}

Processes involving simultaneous wetting and drying have significant differences from those involving only drying. Design, scale-up and control present specific challenges, and operating windows are often narrower. These effects can now be modelled successfully.

\section{References}

[1] Pandey, P. and Bharadwaj, R., eds. Predictive Modeling of Pharmaceutical Unit Operations", 1st Edition. Woodhead Publishing (Elsevier), 2016.

[2] Lyngberg, O., Bijnens, L., Geens, J., Marchut, A., Mehrman, S., Schafer, E. Applications of Modeling in Oral Solid Dosage Form Development and Manufacturing. In: Ierapetritou M., Ramachandran R. (eds) Process Simulation and Data Modeling in Solid Oral Drug Development and Manufacture, Methods in Pharmacology and Toxicology. Humana Press, New York, NY, USA, 2016.

[3] Gupta, R. Fluid bed granulation. Chapter 6 in Pandey \& Bharadwaj, ref.[1].

[4] Kemp, I.C., van Millingen, A. and Khaled, H. Development and verification of a novel design space for fluid bed granulation using a mechanistic model. Paper submitted to Pharmaceutical Development and Technology, 2018.

[5] ICH Q8. ICH harmonised tripartite guideline: Pharmaceutical Development, Q8(R2), 2009. ICH (International Conference on Harmonisation of technical requirements for registration of pharmaceuticals for human use). Available online at www.fda.gov.

[6] Kemp, I.C., Iler, L., Waldron, M. and Turnbull, N. Modelling, experimental trials and design space determination for the GEA ConSigma ${ }^{\mathrm{TM}}$ coater. Paper accepted for Drying Technology, 2018. DOI: 10.1080/07373937.2018.1463244. Originally presented at Eurodrying 2017, Liege, Belgium.

[7] van den Ban, S.; Pitt, K.G.; Whiteman, M. Application of a tablet film coating model to define a process-imposed transition boundary for robust film coating. Pharmaceutical Development and Technology, 23:2 (2018), 176-182. DOI: 10.1080/10837450.2017.1384492..

[8] Ebey, G.C. A thermodynamic model for aqueous tablet film coating. Pharmaceutical Technology, 1987; 11(4): 40-50.

[9] Kemp, I.C. Application of mechanistic drying models in pharmaceuticals and other industries. Paper submitted to Drying Technology, 2018. 